Sharif University of Technology
Scientia Iranica
Transactions E: Industrial Engineering
hCIENTIA

\title{
Difference-type-exponential estimators based on dual auxiliary information under simple random sampling
}

\author{
M. Irfan ${ }^{a, *}$, M. Javed ${ }^{a, b}$, and S. Haider Bhatti ${ }^{a}$ \\ a. Department of Statistics, Government College University, Faisalabad, Pakistan. \\ b. Department of Mathematics, Institute of Statistics Zhejiang University, Hangzhou 310027, China. \\ Received 21 May 2019; received in revised form 27 January 2020; accepted 20 April 2020
}

\author{
KEYWORDS \\ Auxiliary variable; \\ Mean square error; \\ Percent absolute \\ relative bias; \\ Percentage relative \\ efficiency; \\ Ranked auxiliary \\ variable.
}

\begin{abstract}
Auxiliary information plays a vital role in parameter selection and estimation to achieve efficient estimates of unknown population parameters. Dual use of auxiliary information, i.e., "original" and "ranked" auxiliary variables, helps increase the efficiency of estimators. In this paper, the performance of difference-type-exponential estimators was proposed and evaluated based on dual auxiliary information for population mean under simple random sampling. Mathematical expressions for the bias and the mean squared error of the proposed estimators were obtained. Three real-life data sets and Monte Carlo simulation studies were carried out for illustration. The results of empirical and simulation studies indicate that the proposed estimators outperformed their counterparts in terms of mean square errors and percentage relative efficiency.
\end{abstract}

(C) 2022 Sharif University of Technology. All rights reserved.

\section{Introduction}

Over the last few decades, survey sampling has evolved into an extensive body of theories, methods, and operations used daily all over the world. It is broadly used in agriculture, business management, demography, economics, education, engineering, industry, medical sciences, political science, social sciences, and many others. In sample surveys, there are many estimators of finite population mean under simple random sampling that rely on auxiliary information. In fact, proper use of auxiliary information in probability sampling results in a considerable reduction in the variance of the estimator of unknown population parameter(s). The existing estimation procedures are based only on

\footnotetext{
*. Corresponding author.

E-mail addresses: mirfan@gcuf.edu.pk (M. Irfan);

mariajaved@gcuf.edu.pk (M.Javed); goodhaider@gmail.com (S. Haider Bhatti)
}

doi: $10.24200 /$ sci. 2020.53592 .3318 the original form of the supplementary information provided by auxiliary variable(s).

Recently, Haq et al. [1] initiated the idea of utilizing additional information of auxiliary variable along with its original information to boost the efficiency of estimators. This additional information is in the form of ranks of the auxiliary variable, called ranked auxiliary variable. This study is motivated to explore more efficient estimators by using the dual auxiliary information. It proposes two difference-type-exponential estimators based on the original and ranked auxiliary information for efficient estimation of population mean under simple random sampling scheme.

Consider a sample of size $n$ drawn using Simple Random Sampling Without Replacement (SRSWOR) scheme from a population of size $N$, for $i=$ $1,2,3, \ldots, N$. Let $x_{i}, y_{i}$, and $r_{x, i}$ denote the observations on the auxiliary variable, study variable, and ranked auxiliary variable, respectively, for the $i$ th unit of the population. Some useful measures are explained in Table 1.

To obtain bias, Mean Square Error (MSE), and 
Table 1. Some useful measures.

\begin{tabular}{|c|c|c|c|}
\hline Measures & Study variable $(y)$ & Auxiliary variable $(x)$ & Ranked auxiliary variable $\left(r_{x}\right)$ \\
\hline $\begin{array}{l}\text { Sample } \\
\text { mean }\end{array}$ & $\bar{y}=n^{-1} \sum_{i=1}^{n} y_{i}$ & $\bar{x}=n^{-1} \sum_{i=1}^{n} x_{i}$ & $\overline{r_{x}}=n^{-1} \sum_{i=1}^{n} r_{x, i}$ \\
\hline $\begin{array}{l}\text { Population } \\
\text { mean }\end{array}$ & $\bar{Y}=N^{-1} \sum_{i=1}^{N} y_{i}$ & $\bar{X}=N^{-1} \sum_{i=1}^{N} x_{i}$ & $\overline{R_{x}}=N^{-1} \sum_{i=1}^{N} r_{x, i}$ \\
\hline $\begin{array}{l}\text { Population } \\
\text { variance }\end{array}$ & $S_{y}^{2}=(N-1)^{-1} \sum_{i=1}^{N}\left(y_{i}-\bar{Y}\right)^{2}$ & $S_{x}^{2}=(N-1)^{-1} \sum_{i=1}^{N}\left(x_{i}-\bar{X}\right)^{2}$ & $S_{r x}^{2}=(N-1)^{-1} \sum_{i=1}^{N}\left(r_{x, i}-\bar{R}_{x}\right)^{2}$ \\
\hline $\begin{array}{l}\text { Population } \\
\text { coefficient } \\
\text { of variation }\end{array}$ & $C_{y}^{2}=\left(\bar{Y}^{2}\right)^{-1} S_{y}^{2}$ & $C_{x}^{2}=\left(\bar{X}^{2}\right)^{-1} S_{x}^{2}$ & $C_{r}^{2}=\left(\bar{R}_{x}^{2}\right)^{-1} S_{r x}^{2}$ \\
\hline
\end{tabular}

minimum MSE of the proposed estimators, the following relative error terms and their expectations are defined:

$$
\begin{aligned}
\xi_{0} & =\frac{\bar{y}-\bar{Y}}{\bar{Y}}, \quad \xi_{1}=\frac{\overline{r_{x}}-\overline{R_{x}}}{\overline{R_{x}}}, \quad \text { and } \\
\xi_{2} & =\frac{\bar{x}-\bar{X}}{\bar{X}},
\end{aligned}
$$

such that:

$$
\begin{aligned}
& E\left(\xi_{0}\right)=E\left(\xi_{1}\right)=E\left(\xi_{2}\right)=0, \\
& E\left(\xi_{0}^{2}\right)=\psi C_{y}^{2}, \quad E\left(\xi_{1}^{2}\right)=\psi C_{r}^{2}, \\
& E\left(\xi_{2}^{2}\right)=\psi C_{x}^{2}, \quad E\left(\xi_{0} \xi_{1}\right)=\psi \rho_{y r_{x}} C_{y} C_{r}, \\
& E\left(\xi_{1} \xi_{2}\right)=\psi \rho_{x r_{x}} C_{x} C_{r}, \quad E\left(\xi_{0} \xi_{2}\right)=\psi \rho_{y x} C_{y} C_{x},
\end{aligned}
$$

where:

$$
\begin{aligned}
& \psi=\left(\frac{1}{n}-\frac{1}{N}\right), \quad \rho_{y x}=\left(S_{y} S_{x}\right)^{-1} S_{y x}, \\
& \rho_{y r_{x}}=\left(S_{y} S_{r_{x}}\right)^{-1} S_{y r_{x}}, \quad \rho_{x r_{x}}=\left(S_{x} S_{r_{x}}\right)^{-1} S_{x r_{x}}, \\
& S_{y x}=(N-1)^{-1} \sum_{i=1}^{N}\left(y_{i}-\bar{Y}\right)\left(x_{i}-\bar{X}\right), \\
& S_{y r_{x}}=(N-1)^{-1} \sum_{i=1}^{N}\left(y_{i}-\bar{Y}\right)\left(r_{x, i}-\bar{R}_{x}\right), \\
& S_{x r_{x}}=(N-1)^{-1} \sum_{i=1}^{N}\left(x_{i}-\bar{X}\right)\left(r_{x, i}-\bar{R}_{x}\right) .
\end{aligned}
$$

Some important expressions used in upcoming sections are defined below:

$$
\begin{aligned}
& R=\frac{\bar{Y}}{\bar{X}}, \quad R=\frac{\bar{X}}{\bar{Y}}, \quad R_{x}=\frac{\overline{R_{x}}}{\bar{Y}}, \\
& \gamma=\frac{\alpha \bar{X}}{\alpha \bar{X}+\beta}, \quad \kappa=\rho_{y x} \frac{C_{y}}{C_{x}}, \quad \varphi_{1}=\psi C_{x}^{2}, \\
& \varphi_{2}=1+\psi C_{y}^{2}, \quad \varphi_{3}=\psi C_{r}^{2}, \quad \varphi_{4}=\psi C_{x}^{2}(\kappa-1), \\
& \varphi_{5}=\psi C_{x}^{2}\left(\kappa-\frac{1}{2}\right), \quad \varphi_{6}=\psi \rho_{y r_{x}} C_{y} C_{r}, \\
& \varphi_{7}=\psi \rho_{x r_{x}} C_{x} C_{r}, \quad \varphi_{8}=\psi C_{x}^{2}(2 \kappa-1), \\
& \varphi_{9}=\frac{\psi C_{x}^{2}}{2} .
\end{aligned}
$$

\section{Traditional and existing exponential-type estimators}

Several authors have used ratio, product, and regression-type estimators to estimate population mean when both study and auxiliary variables are directly observable. Readers are referred to the studies in [2-18], etc., for more details.

This section gives a brief introduction of traditional estimators: unbiased, ratio, product, and regression as well as the well-known exponential-type estimators of population mean under simple random sampling.

Commonly used unbiased, ratio, product, and regression estimators of the population mean $\bar{Y}$ are as follows:

$$
\widehat{\bar{Y}}=\bar{y}
$$

$$
\widehat{Y_{R}}=\bar{y}\left(\frac{\bar{X}}{\bar{x}}\right), \quad \bar{x} \neq 0,
$$




$$
\begin{aligned}
& \widehat{\overline{Y_{P}}}=\bar{y}\left(\frac{\bar{x}}{\bar{X}}\right), \\
& \widehat{\bar{Y}}_{R E G}=\bar{y}+b(\bar{X}-\bar{x}),
\end{aligned}
$$

where:

$$
b=\frac{\rho_{y x} S_{y}}{S_{x}}
$$

is the slope coefficient.

The expressions for the biases of $\widehat{Y_{R}}$ and $\overline{Y_{P}}$ are given by:

$$
\begin{aligned}
& \operatorname{Bias}\left(\widehat{\overline{Y_{R}}}\right) \cong \psi \bar{Y} C_{x}\left(C_{x}-\rho_{y x} C_{y}\right), \\
& \operatorname{Bias}\left(\widehat{Y_{P}}\right) \cong \psi \bar{Y} C_{x}\left(C_{x}+\rho_{y x} C_{y}\right) .
\end{aligned}
$$

The following ratio and product exponential-type estimators were suggested by Bahl and Tuteja [19]:

$$
\begin{aligned}
& \widehat{\bar{Y}}_{B T, R}=\bar{y} \exp \left(\frac{\bar{X}-\bar{x}}{\bar{X}+\bar{x}}\right), \\
& \widehat{\bar{Y}}_{B T, P}=\bar{y} \exp \left(\frac{\bar{x}-\bar{X}}{\bar{x}+\bar{X}}\right) .
\end{aligned}
$$

Average of Eq. (8) and Eq. (9) can be written as follows:

$$
\widehat{\bar{Y}}_{B T, A v g}=\frac{\bar{y}}{2}\left[\exp \left(\frac{\bar{X}-\bar{x}}{\bar{X}+\bar{x}}\right)+\exp \left(\frac{\bar{x}-\bar{X}}{\bar{x}+\bar{X}}\right)\right] .
$$

Haq and Shabbir [8] proposed three improved exponential-type estimators based on original auxiliary information given by:

$$
\begin{aligned}
\widehat{\bar{Y}}_{H S 1}= & {\left[\frac{\lambda_{1}}{2} \bar{y}\left(\frac{\bar{X}}{\bar{x}}+\frac{\bar{x}}{\bar{X}}\right)+\lambda_{2}(\bar{X}-\bar{x})\right] } \\
& \exp \left(\frac{\bar{X}-\bar{x}}{\bar{X}+\bar{x}}\right) \cdot \\
\widehat{\bar{Y}}_{H S 2}= & {\left[\lambda_{3} \widehat{\bar{Y}}_{B T, A v g}+\lambda_{4}(\bar{X}-\bar{x})\right] } \\
& \exp \left(\frac{\bar{X}-\bar{x}}{\bar{X}+\bar{x}}\right) \cdot \\
\widehat{\bar{Y}}_{H S 3}= & {\left[\frac{\lambda_{5}}{2} \widehat{\bar{Y}}_{B T, A v g}\left(\frac{\bar{X}}{\bar{x}}+\frac{\bar{x}}{\bar{X}}\right)+\lambda_{6}(\bar{X}-\bar{x})\right] } \\
& \exp \left(\frac{\bar{X}-\bar{x}}{\bar{X}+\bar{x}}\right) \cdot
\end{aligned}
$$

Expressions for biases of $\widehat{\bar{Y}}_{H S 1}, \widehat{\bar{Y}}_{H S 2}$, and $\widehat{\bar{Y}}_{H S 3}$ are given by:

$$
\begin{array}{r}
\operatorname{Bias}\left(\widehat{\bar{Y}}_{H S 1}\right) \cong \frac{1}{8}\left[-8 \bar{Y}+\bar{Y}\left\{8+\psi C_{x}\right.\right. \\
\left.\left.\left(7 C_{x}-4 \rho_{y x} C_{y}\right)\right\} \lambda_{1}+4 \bar{X} \psi C_{x}^{2} \lambda_{2}\right], \\
\operatorname{Bias}\left(\widehat{\bar{Y}}_{H S 2}\right) \cong \frac{1}{2}\left[-2 \bar{Y}+\bar{Y}\left\{2+\psi C_{x}\right.\right. \\
\left.\left.\left(C_{x}-\rho_{y x} C_{y}\right)\right\} \lambda_{3}+\bar{X} \psi C_{x}^{2} \lambda_{4}\right], \\
\operatorname{Bias}\left(\widehat{\bar{Y}}_{H S 3}\right) \cong \frac{1}{2}\left[-2 \bar{Y}+\bar{Y}\left\{2+\psi C_{x}\right.\right. \\
\left.\left.\left(2 C_{x}-\rho_{y x} C_{y}\right)\right\} \lambda_{5}+\bar{X} \psi C_{x}^{2} \lambda_{6}\right] .
\end{array}
$$

Ekpenyong and Enang [10] proposed the following two efficient exponential ratio estimators:

$$
\begin{aligned}
& \widehat{\bar{Y}}_{J I 1}=\lambda_{7} \bar{y}+\lambda_{8}(\bar{X}-\bar{x}) \exp \left(\frac{\bar{X}-\bar{x}}{\bar{X}+\bar{x}}\right), \\
& \widehat{\bar{Y}}_{J I 2}=\lambda_{9} \bar{y}+\lambda_{10}(\bar{X}-\bar{x}) \exp \left(\frac{2(\bar{X}-\bar{x})}{\bar{X}+\bar{x}}\right) .
\end{aligned}
$$

Biases of $\widehat{\bar{Y}}_{J I 1}$ and $\widehat{\bar{Y}}_{J I 2}$ are given below:

$$
\begin{aligned}
& \operatorname{Bias}\left(\widehat{\bar{Y}}_{J I 1}\right) \cong \bar{Y}\left[\left(\lambda_{7}-1\right)+\lambda_{8} \stackrel{\prime}{R} \psi \frac{C_{x}^{2}}{2}\right], \\
& \operatorname{Bias}\left(\widehat{\bar{Y}}_{J I 2}\right) \cong \bar{Y}\left[\left(\lambda_{9}-1\right)+\lambda_{10} \stackrel{\prime}{R} \psi C_{x}^{2}\right] .
\end{aligned}
$$

Haq et al. [1] suggested an improved class of estimators following the lines of Shabbir and Gupta [20] and Grover and Kaur [5,6]. This class is based on the original and ranked auxiliary information.

$$
\begin{aligned}
\widehat{\bar{Y}}_{H A}= & {\left[\lambda_{11} \bar{y}+\lambda_{12}(\bar{X}-\bar{x})+\lambda_{13}\left(\bar{R}_{x}-\bar{r}_{x}\right)\right] } \\
& \exp \left(\frac{\alpha(\bar{X}-\bar{x})}{\alpha(\bar{X}-\bar{x})+2 \beta}\right)
\end{aligned}
$$

where $\alpha$ and $\beta$ may be any constant values or functions of the known parameters of the auxiliary variable.

Expression for bias of $\widehat{\bar{Y}}_{H A}$ is stated as follows:

$$
\begin{aligned}
\operatorname{Bias}\left(\widehat{\bar{Y}}_{H A}\right) \cong & \frac{1}{8}\left[-8 \bar{Y}+4 \psi \gamma C_{x}\left(\bar{X} C_{x} \lambda_{12}+\bar{R}_{x}\right.\right. \\
& \left.C_{r} \lambda_{13} \rho_{y x}\right)+\bar{Y} \lambda_{11}\left\{8+\psi \gamma C_{x}\right. \\
& \left.\left.\left(3 \gamma C_{x}-4 C_{y} \rho_{y x}\right)\right\}\right]
\end{aligned}
$$


Remark 2.1. As is given, $\lambda_{i}, i=1,2, \ldots, 13$ appearing in the above equations are the unknown weights determined such that the MSEs are minimized. Therefore, the optimal values of $\lambda_{i}$ are obtained using the following condition:

$$
\begin{aligned}
& \frac{\partial M S E(*)}{\partial \lambda_{i}}=0 \\
& *=\widehat{\bar{Y}}_{H S 1}, \widehat{\bar{Y}}_{H S 2}, \widehat{\bar{Y}}_{H S 3}, \widehat{\bar{Y}}_{J I 1}, \widehat{\bar{Y}}_{J I 2}, \widehat{\bar{Y}}_{H A} \\
& \quad(i=1,2,3, \cdots, 13) .
\end{aligned}
$$

On finding a solution, we have, equations shown in Box I.

Remark 2.2. MSEs and minimum MSEs at optimal values of $\lambda_{i}, i=1,2, \ldots, 13$ of the estimators presented in Eq. (2) to Eq. (20) are given below:

$$
\begin{aligned}
& \operatorname{MSE}(\widehat{\bar{Y}})=V(\widehat{\bar{Y}})=\psi \bar{Y}^{2} C_{y}^{2}, \\
& \operatorname{MSE}\left(\widehat{\overline{Y_{R}}}\right) \cong \psi \bar{Y}^{2}\left[C_{y}^{2}+C_{x}^{2}-2 \rho_{y x} C_{y} C_{x}\right], \\
& \operatorname{MSE}\left(\widehat{\overline{Y_{P}}}\right) \cong \psi \bar{Y}^{2}\left[C_{y}^{2}+C_{x}^{2}+2 \rho_{y x} C_{y} C_{x}\right], \\
& \operatorname{MSE}\left(\widehat{\bar{Y}}_{R E G}\right) \cong \psi \bar{Y}^{2} C_{y}^{2}\left[1-\rho_{y x}^{2}\right], \\
& \operatorname{MSE}\left(\widehat{\bar{Y}}_{B T, R}\right) \cong \frac{\psi}{4} \bar{Y}^{2}\left[4 C_{y}^{2}+C_{x}^{2}-4 \rho_{y x} C_{y} C_{x}\right] \text {, } \\
& \operatorname{MSE}\left(\widehat{\bar{Y}}_{B T, P}\right) \cong \frac{\psi}{4} \bar{Y}^{2}\left[4 C_{y}^{2}+C_{x}^{2}+4 \rho_{y x} C_{y} C_{x}\right] \\
& M S E_{\min }\left(\widehat{\bar{Y}}_{H S 1}\right)= \\
& \frac{\psi \bar{Y}^{2}\left[-25 \psi C_{x}^{4}+16\left(-1+\rho_{y x}^{2}\right)\left(-4+\psi C_{x}^{2}\right) C_{y}^{2}\right]}{64\left[1+\psi C_{x}^{2}+\psi C_{y}^{2}\left(1-\rho_{y x}^{2}\right)\right]} \\
& M S E_{\min }\left(\widehat{\bar{Y}}_{H S 2}\right)= \\
& \frac{\psi \bar{Y}^{2}\left[-\psi C_{x}^{4}+4\left(-1+\rho_{y x}^{2}\right)\left(-4+\psi C_{x}^{2}\right) C_{y}^{2}\right]}{4\left[4+\psi C_{x}^{2}-4 \psi C_{y}^{2}\left(-1+\rho_{y x}^{2}\right)\right]}, \\
& M S E_{\min }\left(\widehat{\bar{Y}}_{H S 3}\right)= \\
& \frac{\psi \bar{Y}^{2}\left[-9 \psi C_{x}^{4}+4\left(-1+\rho_{y x}^{2}\right)\left(-4+\psi C_{x}^{2}\right) C_{y}^{2}\right]}{4\left[4+5 \psi C_{x}^{2}-4 \psi C_{y}^{2}\left(-1+\rho_{y x}^{2}\right)\right]}, \\
& M S E_{\min }\left(\widehat{\bar{Y}}_{J I 1}\right)=\bar{Y}^{2}\left(1-\frac{\varphi_{1}+2 \varphi_{5} \varphi_{9}+\varphi_{2} \varphi_{9}^{2}}{\varphi_{1} \varphi_{2}-\varphi_{5}^{2}}\right),
\end{aligned}
$$

$$
\begin{aligned}
& M S E_{\min }\left(\widehat{\bar{Y}}_{J I 2}\right)=\bar{Y}^{2}\left(1-\frac{\varphi_{1}+2 \varphi_{1} \varphi_{4}+\varphi_{2} \varphi_{1}^{2}}{\varphi_{1} \varphi_{2}-\varphi_{4}^{2}}\right), \\
& M S E_{\min }\left(\widehat{\bar{Y}}_{H A}\right)= \\
& \frac{\psi \bar{Y}^{2}\left[64 C_{y}^{2}\left(1-R_{y \cdot x r_{x}}^{2}\right)-\psi \gamma^{4} C_{x}^{4}-16 \psi \gamma^{2} C_{x}^{2} C_{y}^{2}\left(1-R_{y \cdot x r_{x}}^{2}\right)\right]}{64\left[1+\psi C_{y}^{2}\left(1-R_{y \cdot x r_{x}}^{2}\right)\right]} .
\end{aligned}
$$

where:

$$
R_{y . x r_{x}}^{2}=\frac{\rho_{y x}^{2}+\rho_{y r_{x}}^{2}-2 \rho_{y x} \rho_{y r_{x}} \rho_{x r_{x}}}{1-\rho_{x r_{x}}^{2}}
$$

\section{Proposed estimators}

In this section, two new difference-type-exponential estimators are proposed for population mean under SRSWOR. These estimators are based on the dual use of auxiliary information. (1) The auxiliary variable uses original/actual measurements of the auxiliary variable; (2) The ranked auxiliary variable uses the ranks of the auxiliary variable. Mathematical properties such as bias, MSE, and minimum MSE of the proposed estimators are derived up to the first order of approximation. The bias of an estimator is the difference between the estimator's expected value and the true value of the parameter being estimated, i.e., $\operatorname{Bias}(\widehat{\bar{Y}})=E(\widehat{\bar{Y}}-\bar{Y})$, and MSE can be defined as the divergence of the estimator values from the true parameter value, i.e., $\operatorname{MSE}(\widehat{\bar{Y}})=E(\widehat{\bar{Y}}-\bar{Y})^{2}$.

\subsection{First proposed estimator}

$$
\begin{aligned}
\widehat{\bar{Y}}_{P 1}= & \frac{\lambda_{14}}{2}\left(\frac{\bar{X}}{\bar{x}}+\frac{\bar{x}}{\bar{X}}\right) \widehat{\bar{Y}}_{B T, A v g}+\lambda_{15}\left(\overline{R_{x}}-\overline{r_{x}}\right) \\
& +\lambda_{16}(\bar{X}-\bar{x}) \exp \left(\frac{\bar{X}-\bar{x}}{\bar{X}+\bar{x}}\right),
\end{aligned}
$$

where $\lambda_{14}, \lambda_{15}$, and $\lambda_{16}$ are the properly chosen constants.

Here, we use Eq. (1) to rewrite the estimator in Eq. (34), subtract $\bar{Y}$ from both sides, and get the expression up to the first order of approximation in this way:

$$
\begin{aligned}
\widehat{\bar{Y}}_{P 1}-\bar{Y}= & {\left[\lambda_{14} \bar{Y}+\frac{5 \lambda_{14} \bar{Y} \xi_{2}^{2}}{8}+\lambda_{14} \bar{Y} \xi_{0}-\lambda_{15} \overline{R_{x}} \xi_{1}\right.} \\
& \left.-\lambda_{16} \bar{X} \xi_{2}+\frac{\lambda_{16} \bar{X} \xi_{2}^{2}}{2}-\bar{Y}\right]
\end{aligned}
$$

Applying expectation on both sides of Eq. (35), we get the bias of the proposed estimator as follows: 


$$
\begin{aligned}
& \operatorname{Bias}\left(\widehat{\bar{Y}}_{H A}\right) \cong \frac{1}{8}\left[-8 \bar{Y}+4 \psi \gamma C_{x}\left(\bar{X} C_{x} \lambda_{12}+\bar{R}_{x} C_{r} \lambda_{13} \rho_{y x}\right)+\bar{Y} \lambda_{11}\left\{8+\psi \gamma C_{x}\left(3 \gamma C_{x}-4 C_{y} \rho_{y x}\right)\right\}\right] \\
& \lambda_{1}=\frac{8+3 \psi C_{x}^{2}}{8\left[1+\psi C_{x}^{2}+\psi C_{y}^{2}\left(1-\rho_{y x}^{2}\right)\right]}, \\
& \lambda_{2}=\frac{\bar{Y}\left[8 C_{y} \rho_{y x}+C_{x}\left\{-4+\psi\left(C_{x}^{2}+3 \rho_{y x} C_{y} C_{x}-4\left(-1+\rho_{y x}^{2}\right) C_{y}^{2}\right)\right\}\right]}{8 \bar{X} C_{x}\left[1+\psi C_{x}^{2}+\psi C_{y}^{2}\left(1-\rho_{y x}^{2}\right)\right]}, \\
& \lambda_{3}=\frac{4}{4+\psi C_{x}^{2}-4 \psi C_{y}^{2}\left(-1+\rho_{y x}^{2}\right)}, \\
& \lambda_{4}=\frac{\bar{Y}}{2 \bar{X}}\left[1+\frac{-8 C_{x}+8 C_{y} \rho_{y x}}{C_{x}\left[4+\psi C_{x}^{2}-4 \psi C_{y}^{2}\left(-1+\rho_{y x}^{2}\right)\right]}\right], \\
& \lambda_{5}=\frac{4+2 \psi C_{x}^{2}}{4+5 \psi C_{x}^{2}-4 \psi C_{y}^{2}\left(-1+\rho_{y x}^{2}\right)}, \\
& \lambda_{6}=\frac{\bar{Y}\left[8 C_{y} \rho_{y x}+C_{x}\left\{-4+\psi\left(C_{x}^{2}+4 \rho_{y x} C_{y} C_{x}-4 C_{y}^{2}\left(-1+\rho_{y x}^{2}\right)\right)\right\}\right]}{2 \bar{X} C_{x}\left[4+5 \psi C_{x}^{2}-4 \psi C_{y}^{2}\left(-1+\rho_{y x}^{2}\right)\right]}, \\
& \lambda_{7}=\frac{\varphi_{1}+\varphi_{5} \varphi_{9}}{\varphi_{1} \varphi_{2}-\varphi_{5}^{2}} \\
& \lambda_{8}=R\left(\frac{\varphi_{5}+\varphi_{2} \varphi_{9}}{\varphi_{1} \varphi_{2}-\varphi_{5}^{2}}\right) \\
& \lambda_{9}=\frac{\varphi_{1}+\varphi_{1} \varphi_{4}}{\varphi_{1} \varphi_{2}-\varphi_{4}^{2}} \\
& \lambda_{10}=R\left(\frac{\varphi_{4}+\varphi_{1} \varphi_{2}}{\varphi_{1} \varphi_{2}-\varphi_{4}^{2}}\right) \\
& \lambda_{11}=\frac{8-\psi \gamma^{2} C_{x}^{2}}{8\left[1+\psi C_{y}^{2}\left(1-R_{y . x r_{x}}^{2}\right)\right]}, \\
& \lambda_{12}=\frac{\bar{Y}\left[\psi \gamma^{3} C_{x}^{3}\left(-1+\rho_{x r_{x}}^{2}\right)+\left(-8 C_{y}+\psi \gamma^{2} C_{x}^{2} C_{y}\right)\left(\rho_{y x}-\rho_{x r_{x}} \rho_{y r_{x}}\right)+4 \gamma C_{x}\left(-1+\rho_{x r_{x}}^{2}\right)\left\{-1+\psi C_{y}^{2}\left(\left(1-R_{y . x r_{x}}^{2}\right)\right)\right\}\right]}{8 \bar{X} C_{x}\left(-1+\rho_{x r_{x}}^{2}\right)\left[1+\psi C_{y}^{2}\left(1-R_{y . x r_{x}}^{2}\right)\right]}, \\
& \lambda_{13}=\frac{\bar{Y}\left(8-\psi \gamma^{2} C_{x}^{2}\right) C_{y}\left(\rho_{x r_{x}} \rho_{y x}-\rho_{y r_{x}}\right)}{8 \overline{R_{x}} C_{r}\left(-1+\rho_{x r_{x}}^{2}\right)\left[1+\psi C_{y}^{2}\left(1-R_{y . x r_{x}}^{2}\right)\right]} .
\end{aligned}
$$




$$
\operatorname{Bias}\left(\widehat{\bar{Y}}_{P 1}\right) \cong\left(\lambda_{14}-1\right) \bar{Y}+\frac{\varphi_{1}}{2}\left(\lambda_{16} \bar{X}+\frac{5 \lambda_{14} \bar{Y}}{4}\right)
$$

Squaring both sides of Eq. (35) up to the first order of approximation, we have:

$$
\begin{aligned}
& \left(\widehat{\bar{Y}}_{P 1}-\bar{Y}\right)^{2} \cong\left[\bar{Y}^{2}+\lambda_{14}^{2} \bar{Y}^{2}+\lambda_{14}^{2} \bar{Y}^{2} \xi_{0}^{2}+\lambda_{15}^{2} \bar{R}_{x}^{2} \xi_{1}^{2}\right. \\
& +\lambda_{16}^{2} \bar{X}^{2} \xi_{2}^{2}+\frac{5}{4} \lambda_{14}^{2} \bar{Y}^{2} \xi_{2}^{2}+\lambda_{14} \lambda_{16} \overline{X Y} \xi_{2}^{2}-2 \lambda_{14} \bar{Y}^{2} \\
& -\frac{5}{4} \lambda_{14} \bar{Y}^{2} \xi_{2}^{2}-2 \lambda_{14} \lambda_{15} \overline{Y R_{x}} \xi_{0} \xi_{1}-2 \lambda_{14} \lambda_{16} \overline{X Y} \xi_{0} \xi_{2} \\
& \left.+2 \lambda_{15} \lambda_{16} \overline{X R_{x}} \xi_{1} \xi_{2}-\lambda_{16} \overline{X Y} \xi_{2}^{2}\right] .
\end{aligned}
$$

The MSE of $\widehat{\bar{Y}}_{P 1}$ is obtained by taking expectation of both sides of Eq. (36)

$$
\begin{aligned}
& M S E\left(\widehat{\bar{Y}}_{P 1}\right) \cong \bar{Y}^{2}\left[1+\lambda_{14}^{2} \varphi_{2}+\frac{5}{4} \lambda_{14}^{2} \varphi_{1}+\lambda_{15}^{2} \stackrel{\prime}{R}_{x}^{2} \varphi_{3}\right. \\
& +\lambda_{16}^{2} \stackrel{\prime}{R^{2}} \varphi_{1}-2 \lambda_{14}-\frac{5}{4} \lambda_{14} \varphi_{1}-\lambda_{16} \stackrel{\prime}{R} \varphi_{1} \\
& \left.-2 \lambda_{14} \lambda_{15}{ }^{\prime} R_{x} \varphi_{6}+2 \lambda_{15} \lambda_{16} \stackrel{\prime}{R} R_{x}^{\prime} \varphi_{7}-\lambda_{14} \lambda_{16} \stackrel{\prime}{R} \varphi_{8}\right]{ }_{(38)}
\end{aligned}
$$

Now, we have to choose the weights of $\lambda_{14}, \lambda_{15}$, and $\lambda_{16}$ such that the resulting MSE of $\widehat{\bar{Y}}_{P 1}$ is minimized. Thus, the optimal weights of $\lambda_{14}, \lambda_{15}$ and $\lambda_{16}$ are selected with the help of the following equations.

$$
\begin{aligned}
& \frac{\partial M S E\left(\widehat{\bar{Y}}_{P 1}\right)}{\partial \lambda_{14}}=\left(8 \varphi_{2}+10 \varphi_{1}\right) \lambda_{14}-8 \lambda_{15} \stackrel{\prime}{R}_{x} \varphi_{6} \\
& -4 \lambda_{16} \stackrel{\prime}{R} \varphi_{8}-8-5 \varphi_{1}, \\
& \frac{\partial M S E\left(\widehat{\bar{Y}}_{P 1}\right)}{\partial \lambda_{15}}=2 \lambda_{15} \stackrel{\prime}{R}_{x}^{2} \varphi_{3}-2 \lambda_{14} R_{x}^{\prime} \varphi_{6} \\
& +2 \lambda_{16} \stackrel{\prime}{R} \stackrel{\prime}{R}_{x} \varphi_{7}, \\
& \frac{\partial M S E\left(\widehat{\bar{Y}}_{P 1}\right)}{\partial \lambda_{16}}=2 \lambda_{16} R^{2} \varphi_{1}+2 \lambda_{15} \stackrel{\prime}{R}^{\prime} R_{x} \varphi_{7} \\
& -\lambda_{14} \stackrel{\prime}{R} \varphi_{8}-\stackrel{\prime}{R} \varphi_{1} .
\end{aligned}
$$

Setting $\frac{\partial M S E\left(\hat{\bar{Y}}_{P 1}\right)}{\partial \lambda_{i}}=0, i=14,15,16$ and solving simultaneously, we get:

$\lambda_{14(\text { opt })}=\frac{E_{1} E_{2}-2 E_{3} \varphi_{1} \varphi_{3}}{E_{2} E_{4}-2 E_{3}^{2}}$,

$\lambda_{15(\text { opt })}=\frac{2 \varphi_{6}\left(E_{1} E_{2}-2 E_{3} \varphi_{1} \varphi_{3}\right)+\varphi_{7}\left(E_{1} E_{3}-\varphi_{1} \varphi_{3} E_{4}\right)}{2 \varphi_{3} R_{x}{ }^{\prime}\left(E_{2} E_{4}-2 E_{3}^{2}\right)}$,

$$
\lambda_{16(o p t)}=\frac{\varphi_{1} \varphi_{3} E_{4}-E_{1} E_{3}}{2 R^{\prime}\left(E_{2} E_{4}-2 E_{3}^{2}\right)},
$$

where:

$$
\begin{aligned}
& E_{1}=8 \varphi_{3}+5 \varphi_{1} \varphi_{3}, \quad E_{2}=\varphi_{1} \varphi_{3}-\varphi_{7}^{2} \\
& E_{3}=2 \varphi_{6} \varphi_{7}-\varphi_{3} \varphi_{8}, \quad E_{4}=8 \varphi_{2} \varphi_{3}+10 \varphi_{1} \varphi_{3}-8 \varphi_{6}^{2} .
\end{aligned}
$$

Inserting optimal weights of $\lambda_{14}, \lambda_{15}$, and $\lambda_{16}$ in Eq. (38), we get the minimum MSE of the proposed estimator as follows:

$$
\begin{aligned}
& M S E_{\min }\left(\widehat{\bar{Y}}_{P 1}\right)=\frac{\bar{Y}^{2}}{4 \varphi_{3} F_{1}^{2}} \\
& {\left[4 \varphi_{3} F_{1}^{2}+\left(4 \varphi_{2} \varphi_{3}-4 \varphi_{6}^{2}+5 \varphi_{1} \varphi_{3}\right)\right.} \\
& F_{2}^{2}+\left(\varphi_{1} \varphi_{3}-\varphi_{7}^{2}\right) F_{3}^{2}-\left(8+5 \varphi_{1}\right) \varphi_{3} F_{1} F_{2} \\
& \left.+2\left(2 \varphi_{6} \varphi_{7}-\varphi_{3} \varphi_{8}\right) F_{2} F_{3}-2 \varphi_{1} \varphi_{3} F_{1} F_{3}\right]
\end{aligned}
$$

where:

$$
\begin{aligned}
& F_{1}=E_{2} E_{4}-2 E_{3}^{2}, \\
& F_{2}=E_{1} E_{2}-2 \varphi_{1} \varphi_{3} E_{3}, \\
& F_{3}=\varphi_{1} \varphi_{3} E_{4}-E_{1} E_{3} .
\end{aligned}
$$

\subsection{Second proposed estimator}

$$
\begin{aligned}
\widehat{\bar{Y}}_{P 2}= & \lambda_{17} \bar{y}+\lambda_{18}\left(\overline{R_{x}}-\overline{r_{x}}\right) \\
& +\lambda_{19}(\bar{X}-\bar{x}) \exp \left(\frac{2(\bar{X}-\bar{x})}{\bar{X}+\bar{x}}\right),
\end{aligned}
$$

where $\lambda_{17}, \lambda_{18}$, and $\lambda_{19}$ are the properly chosen constants.

Following the same procedure mentioned in Section 3.1, we have the following expressions:

$$
\begin{aligned}
& \widehat{\bar{Y}}_{P 2}-\bar{Y}= \\
& {\left[\lambda_{17} \bar{Y}+\lambda_{17} \bar{Y} \xi_{0}-\lambda_{18} \overline{R_{x}} \xi_{1}-\lambda_{19} \bar{X} \xi_{2}+\lambda_{19} \bar{X} \xi_{2}^{2}-\bar{Y}\right]} \\
& \operatorname{Bias}\left(\widehat{\bar{Y}}_{P 2}\right) \cong \bar{Y}\left[\left(\lambda_{17}-1\right)+\lambda_{19} \varphi_{1} \stackrel{\prime}{R}\right] \\
& \left(\widehat{\bar{Y}}_{P 2}-\bar{Y}\right)^{2} \cong\left[\bar{Y}^{2}+\lambda_{17}^{2} \bar{Y}^{2}+\lambda_{17}^{2} \bar{Y}^{2} \xi_{0}^{2}+\lambda_{18}^{2} \bar{R}_{x}^{2} \xi_{1}^{2}\right. \\
& \quad+\lambda_{19}^{2} \bar{X}^{2} \xi_{2}^{2}+2 \lambda_{17} \lambda_{19} \overline{X Y} \xi_{2}^{2}-2 \lambda_{17} \bar{Y}^{2} \\
& \quad-2 \lambda_{17} \lambda_{18} \overline{Y R_{x}} \xi_{0} \xi_{1}-2 \lambda_{17} \lambda_{19} \overline{X Y} \xi_{0} \xi_{2} \\
& \left.\quad+2 \lambda_{18} \lambda_{19} \overline{X R_{x}} \xi_{1} \xi_{2}-2 \lambda_{19} \overline{X Y} \xi_{2}^{2}\right]
\end{aligned}
$$




$$
\begin{aligned}
& \operatorname{MSE}\left(\widehat{\bar{Y}}_{P 2}\right) \cong \bar{Y}^{2}
\end{aligned}
$$

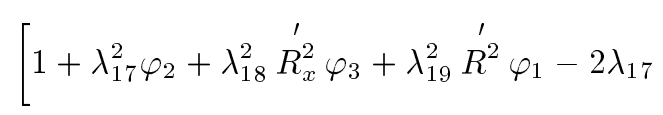

$$
\begin{aligned}
& -2 \lambda_{19} \stackrel{\prime}{R} \varphi_{1}+2 \lambda_{18} \lambda_{19} \stackrel{\prime}{R} \stackrel{\prime}{R}{ }_{x} \varphi_{7} \\
& \left.-2 \lambda_{17} \lambda_{18} \stackrel{\prime}{R}_{x} \varphi_{6}-2 \lambda_{17} \lambda_{19} \stackrel{\prime}{R} \varphi_{4}\right]
\end{aligned}
$$

The optimal weights of $\lambda_{17}, \lambda_{18}$, and $\lambda_{19}$ are obtained as follows:

$$
\begin{aligned}
& \frac{\partial M S E\left(\widehat{\bar{Y}}_{P 2}\right)}{\partial \lambda_{17}} \\
& =2\left(\lambda_{17} \varphi_{2}-1-\lambda_{19} \stackrel{\prime}{R} \varphi_{4}-\lambda_{18} \stackrel{\prime}{R} \varphi_{6}\right), \\
& \frac{\partial M S E\left(\widehat{\bar{Y}}_{P 2}\right)}{\partial \lambda_{18}} \\
& =2\left(\lambda_{18} \stackrel{\prime}{R_{x}^{2}} \varphi_{3}+\lambda_{19} \stackrel{\prime}{R} \stackrel{\prime}{R} \varphi_{x}-\lambda_{17} \stackrel{\prime}{R} \varphi_{6}\right), \\
& \frac{\partial M S E\left(\widehat{\bar{Y}}_{P 2}\right)}{\partial \lambda_{19}}= \\
& 2\left(\lambda_{19} \stackrel{\prime}{R^{2}} \varphi_{1}+\lambda_{18} \stackrel{\prime}{R} \stackrel{\prime}{R} \varphi_{7}-\stackrel{\prime}{R} \varphi_{1}-\lambda_{17} \stackrel{\prime}{R} \varphi_{4}\right) .
\end{aligned}
$$

Setting $\frac{\partial M S E\left(\hat{\bar{Y}}_{P 2}\right)}{\partial \lambda_{i}}=0, i=17,18,19$ and solving simultaneously, we get:

$$
\begin{aligned}
& \lambda_{17(\text { opt })}=\frac{\varphi_{2} \varphi_{3} E_{5}-\left(\varphi_{2} \varphi_{7}+\varphi_{6} E_{7}\right) E_{6}+\varphi_{4} E_{7} E_{8}}{\varphi_{2}\left(E_{5} E_{8}-E_{6}^{2}\right)}, \\
& \lambda_{18(\text { opt })}=\frac{\varphi_{6} E_{5}-E_{6} E_{7}}{R_{x}^{\prime}\left(E_{5} E_{8}-E_{6}^{2}\right)}, \\
& \lambda_{19(\text { opt })}=\frac{E_{7} E_{8}-\varphi_{6} E_{6}}{R^{\prime}\left(E_{5} E_{8}-E_{6}^{2}\right)},
\end{aligned}
$$

where:

$$
\begin{array}{ll}
E_{5}=\varphi_{1} \varphi_{2}-\varphi_{4}^{2}, & E_{6}=\varphi_{2} \varphi_{7}-\varphi_{4} \varphi_{6}, \\
E_{7}=\varphi_{1} \varphi_{2}+\varphi_{4}, & E_{8}=\varphi_{2} \varphi_{3}-\varphi_{6}^{2} .
\end{array}
$$

Inserting optimal weights of $\lambda_{17}, \lambda_{18}$, and $\lambda_{19}$ in Eq. (44), we get the minimum MSE of the proposed estimator as follows:

$$
M S E_{\min }\left(\widehat{\bar{Y}}_{P 2}\right)=\frac{\bar{Y}^{2}}{\varphi_{2} F_{4}^{2}}\left[\varphi_{2} F_{4}^{2}+\varphi_{1} \varphi_{2} F_{5}^{2}+\varphi_{2} \varphi_{3} F_{6}^{2}\right.
$$

$$
\begin{aligned}
& +F_{7}^{2}-2\left(\varphi_{1} F_{4}-\varphi_{7} F_{6}\right) \varphi_{2} F_{5} \\
& \left.-2\left(\varphi_{6} F_{6}+\varphi_{4} F_{5}+F_{4}\right) F_{7}\right],
\end{aligned}
$$

where:

$$
\begin{aligned}
& F_{4}=E_{5} E_{8}-E_{6}^{2}, \quad F_{5}=E_{7} E_{8}-\varphi_{6} E_{6}, \\
& F_{6}=\varphi_{6} E_{5}-E_{6} E_{7}, \\
& F_{7}=\varphi_{2} \varphi_{3} E_{5}-\varphi_{2} \varphi_{7} E_{6}-\varphi_{6} E_{6} E_{7}+\varphi_{4} E_{7} E_{8} .
\end{aligned}
$$

Remark 3.1. Of note, the parameters $\rho_{y x}, \rho_{y r_{x}}$, $\rho_{x r_{x}}, C_{y}, C_{x}$, and $C_{r}$ appearing in the expressions of optimal weights and the minimum MSEs are generally unknown. However, these parameters can be estimated quite accurately from the preliminary data or from the repeated surveys based on sampling over several occasions. Many different authors have dealt with utilization of prior information of parameters at the estimation stage including Singh and Singh [21] and Vishwakarma and Kumar [22].

\section{Applications}

In this section, three real-life datasets are used to evaluate the performance of the proposed estimators as compared to the existing estimators in terms of Percentage Absolute Relative Bias (PARB), MSE, and Percentage Relative Efficiencies (PRE). For more details of these measures, see Rao et al. [23], Silva and Skinner [24], Nidhi et al. [25], etc. MSEs are calculated using the expressions defined in Sections 2 and 3. PARB and PRE of an estimator can be computed through the following expressions:

$$
\begin{aligned}
& P A R B(*)=\left|\frac{(*)-\bar{Y}}{\bar{Y}}\right| \times 100, \\
& P R E=\frac{M S E(\widehat{\bar{Y}})}{M S E(*)} \times 100,
\end{aligned}
$$

where $*=\widehat{\bar{Y}}, \widehat{\bar{Y}}_{R E G}, \widehat{\bar{Y}}_{H S 1}, \widehat{\bar{Y}}_{H S 2}, \widehat{\bar{Y}}_{H S 3}, \widehat{\bar{Y}}_{J I 1}, \widehat{\bar{Y}}_{J I 2}$, $\widehat{\bar{Y}}_{H A}, \widehat{\bar{Y}}_{P 1}, \widehat{\bar{Y}}_{P 2}$.

Population 1 [26].

$$
\begin{aligned}
& N=69, \quad n=12, \quad \bar{Y}=135.2608, \quad \bar{X}=345.7536, \\
& C_{y}=0.8422, \quad C_{x}=0.8479, \quad C_{r}=0.5747, \\
& \beta_{2(x)}=7.2159, \quad \bar{R}_{x}=34.9565, \quad \rho_{y x}=0.9224, \\
& \rho_{y r_{x}}=0.7136, \quad \rho_{x r_{x}}=0.8185 .
\end{aligned}
$$

Population 2 [10]. 


$$
\begin{aligned}
& N=923, \quad n=180, \quad \bar{Y}=436.4345, \quad \bar{X}=11440.5, \\
& C_{y}=1.7183, \quad C_{x}=1.8645, \quad C_{r}=0.577, \\
& \beta_{2(x)}=18.7208, \quad \bar{R}_{x}=461.9642, \quad \rho_{y x}=0.9543, \\
& \rho_{y r_{x}}=0.6442, \quad \rho_{x r_{x}}=0.6306 .
\end{aligned}
$$

\section{Population 3 [27].}

$$
\begin{aligned}
& N=854, \quad n=290, \quad \bar{Y}=2930.12, \\
& \bar{X}=37600.11, \quad C_{y}=5.8379, \quad C_{x}=3.8509, \\
& C_{r}=0.1883, \quad \beta_{2(x)}=312.0651 \\
& \bar{R}_{x}=426.8747, \quad \rho_{y x}=0.9165 \\
& \rho_{y r_{x}}=0.2585, \quad \rho_{x r_{x}}=0.3458 .
\end{aligned}
$$

Table 2 shows the PARB of all the estimators; and Table 3 gives the MSEs and PREs.

\section{Simulation study based on real data sets}

Monte Carlo simulation study is carried out to check the potential of the proposed estimators over the competing estimators through $\mathrm{R}$ software.
A step-by-step approach to the simulation study is as follows:

1. Select an SRSWOR of size $n$ from the population of size $N$;

2. Use sample data from Step 1 to find PARB and MSE of all the estimators under study;

3. Repeat 50,000 times Steps 1 and 2;

4. Obtain 50,000 values for PARB and MSE of all the estimators;

5. Average of 50,000 values obtained in Step 4 in the PARB and MSE;

6. Calculate PARB and PREs (with respect to sample mean $\widehat{\bar{Y}}$ ) of all the estimators.

\section{Important findings}

- From Table 2, the PARB of the proposed estimators is the least among all the competing estimators for all three data sets;

- From Table 3, it is imperative to mention that the proposed estimators $\widehat{\bar{Y}}_{P 1}$ and $\widehat{\bar{Y}}_{P 2}$ have minimum MSEs and maximum PREs compared to all the traditional and existing estimators in all populations;

- From Table 4, simulation study indicates that PARB of the proposed estimators is lower than the com-

Table 2. Numerical comparison of percentage absolute relative bias.

\begin{tabular}{lccc}
\hline Estimators & Population 1 & Population 2 & Population 3 \\
\hline$\widehat{\bar{Y}}$ & - & - & - \\
$\widehat{\bar{Y}}_{R E G}$ & - & - & - \\
$\widehat{\bar{Y}}_{H S 1}$ & 0.5901 & 0.1062 & 1.1345 \\
$\widehat{\bar{Y}}_{H S 2}$ & 0.6905 & 0.1153 & 1.1993 \\
$\widehat{\bar{Y}}_{H S 3}$ & 0.5439 & 0.1017 & 1.1069 \\
$\widehat{\bar{Y}}_{J I 1}$ & 0.6399 & 0.1115 & 1.1195 \\
$\widehat{\bar{Y}}_{J I 2}$ & 0.4642 & 0.0961 & 0.9136 \\
$\widehat{\bar{Y}}_{H A}^{(1)}\left(\alpha=1, \beta=C_{x}\right)$ & 0.6857 & 0.1129 & 1.1855 \\
$\widehat{\bar{Y}}_{H A}^{(2)}\left(\alpha=1, \beta=\beta_{2(x)}\right)$ & 0.6863 & 0.1130 & 1.1857 \\
$\widehat{\bar{Y}}_{H A}^{(3)}\left(\alpha=\beta_{2(x)}, \beta=C_{x}\right)$ & 0.6857 & 0.1129 & 1.1855 \\
$\widehat{\bar{Y}}_{H A}^{(4)}\left(\alpha=C_{x}, \beta=\beta_{2(x)}\right)$ & 0.6865 & 0.1130 & 1.1856 \\
$\widehat{\bar{Y}}_{H A}^{(5)}\left(\alpha=1, \beta=\rho_{y x}\right)$ & 0.6857 & 0.1130 & 1.1855 \\
$\widehat{\widehat{Y}}_{H A}^{(6)}\left(\alpha=C_{x}, \beta=\rho_{y x}\right)$ & 0.6858 & 0.1129 & 1.1855 \\
$\widehat{\bar{Y}}_{H A}^{(7)}\left(\alpha=\rho_{y x}, \beta=C_{x}\right)$ & 0.6857 & 0.1129 & 1.1855 \\
$\widehat{\bar{Y}}_{H A}^{(8)}\left(\alpha=\beta_{2(x)}, \beta=\rho_{y x}\right)$ & 0.6857 & 0.1130 & 1.1855 \\
$\widehat{\bar{Y}}_{H A}^{(9)}\left(\alpha=\rho_{y x}, \beta=\beta_{2(x)}\right)$ & 0.6863 & 0.1129 & 1.1858 \\
$\widehat{\bar{Y}}_{H A}^{(10)}(\alpha=1, \beta=N \bar{X})$ & 0.6981 & 0.1138 & 1.1974 \\
$\widehat{\bar{Y}}_{P 1}$ & 0.3343 & 0.0845 & 0.9117 \\
$\widehat{\bar{Y}}_{P 2}(\alpha)$ & 0.3588 & 0938 & 0.8730 \\
\hline
\end{tabular}


Table 3. Mean Square Error (MSE) and Percentage Relative Efficiencies (PRE) of the existing and proposed estimators.

\begin{tabular}{|c|c|c|c|c|c|c|}
\hline \multirow[b]{2}{*}{ Estimators } & \multicolumn{2}{|c|}{ Population 1} & \multicolumn{2}{|c|}{ Population 2} & \multicolumn{2}{|c|}{ Population 3} \\
\hline & MSE's & PRE's & MSE's & PRE's & MSE's & PRE's \\
\hline$\widehat{\bar{Y}}$ & 893.344 & 100.000 & 2515.074 & 100.000 & 666353.000 & 100.000 \\
\hline$\widehat{\bar{Y}}_{R E G}$ & 133.267 & 670.342 & 224.611 & 1119.747 & 106635.000 & 624.891 \\
\hline$\widehat{\bar{Y}}_{H S 1}$ & 107.982 & 827.308 & 202.369 & 1242.816 & 97410.320 & 684.068 \\
\hline$\widehat{\bar{Y}}_{H S 2}$ & 126.334 & 707.129 & 219.747 & 1144.532 & 102974.400 & 647.105 \\
\hline$\widehat{\bar{Y}}_{H S 3}$ & 99.528 & 897.581 & 193.846 & 1297.460 & 95034.750 & 701.168 \\
\hline$\widehat{\bar{Y}}_{J I 1}$ & 117.484 & 760.396 & 212.505 & 1183.536 & 96116.890 & 693.274 \\
\hline$\widehat{\bar{Y}}_{J I 2}$ & 84.936 & 1051.785 & 183.133 & 1373.359 & 78444.970 & 849.453 \\
\hline$\widehat{\bar{Y}}_{H A}^{(1)}$ & 125.473 & 711.981 & 215.232 & 1168.541 & 101787.500 & 654.651 \\
\hline$\widehat{\bar{Y}}_{H A}^{(2)}$ & 125.577 & 711.391 & 215.239 & 1168.503 & 101806.400 & 654.529 \\
\hline$\widehat{\bar{Y}}_{H A}^{(3)}$ & 125.460 & 712.055 & 215.231 & 1168.546 & 101787.300 & 654.652 \\
\hline$\widehat{\bar{Y}}_{H A}^{(4)}$ & 125.597 & 711.278 & 215.235 & 1168.525 & 101792.300 & 654.620 \\
\hline$\widehat{\bar{Y}}_{H A}^{(5)}$ & 125.474 & 711.975 & 215.232 & 1168.541 & 101787.300 & 654.652 \\
\hline$\widehat{\bar{Y}}_{H A}^{(6)}$ & 125.477 & 711.958 & 215.232 & 1168.541 & 101787.300 & 654.652 \\
\hline$\widehat{\bar{Y}}_{H A}^{(7)}$ & 125.474 & 711.975 & 215.232 & 1168.541 & 101787.500 & 654.651 \\
\hline$\widehat{\bar{Y}}_{H A}^{(8)}$ & 125.460 & 712.055 & 215.231 & 1168.546 & 101787.300 & 654.652 \\
\hline$\widehat{\bar{Y}}_{H A}^{(9)}$ & 125.587 & 711.335 & 215.239 & 1168.503 & 101808.100 & 654.519 \\
\hline$\widehat{\bar{Y}}_{H A}^{(10)}$ & 127.734 & 699.378 & 216.793 & 1160.127 & 102806.400 & 648.162 \\
\hline$\widehat{\bar{Y}}_{P 1}$ & 61.170 & 1460.428 & 161.068 & 1561.498 & 78281.210 & 851.229 \\
\hline$\widehat{\bar{Y}}_{P 2}$ & 65.656 & 1360.643 & 178.828 & 1406.421 & 74957.120 & 888.979 \\
\hline
\end{tabular}

Table 4. Percentage absolute relative bias of estimators with respect to $\widehat{\bar{Y}}$ based on simulation study.

\begin{tabular}{|c|c|c|c|c|c|c|c|c|c|}
\hline \multirow[b]{2}{*}{ Estimators } & \multicolumn{3}{|c|}{ Population 1} & \multicolumn{3}{|c|}{ Population 2} & \multicolumn{3}{|c|}{ Population 3} \\
\hline & $n=12$ & $n=14$ & $n=16$ & $n=180$ & $n=200$ & $n=230$ & $n=290$ & $n=310$ & $n=330$ \\
\hline$\widehat{\bar{Y}}$ & - & - & - & - & - & - & - & - & - \\
\hline$\widehat{\bar{Y}}_{R E G}$ & - & - & - & - & - & - & - & - & - \\
\hline $\bar{Y}_{H S 1}$ & 0.5140 & 0.4522 & 0.3982 & 0.0967 & 0.0873 & 0.0747 & 0.9971 & 0.9283 & 0.8595 \\
\hline$\overline{\bar{Y}}_{H S 2}$ & 0.6106 & 0.5190 & 0.4469 & 0.1056 & 0.0942 & 0.0795 & 1.0573 & 0.9771 & 0.8997 \\
\hline$\widehat{\widehat{Y}}_{H S 3}$ & 0.4688 & 0.4207 & 0.3753 & 0.0923 & 0.0839 & 0.0723 & 0.9703 & 0.9065 & 0.8415 \\
\hline$\widehat{\widehat{Y}}_{J I 1}$ & 0.5639 & 0.4870 & 0.4236 & 0.1019 & 0.0913 & 0.0775 & 0.9871 & 0.9196 & 0.8522 \\
\hline$\widehat{\bar{Y}}_{J I 2}$ & 0.3864 & 0.3654 & 0.3356 & 0.0867 & 0.0796 & 0.0694 & 0.7930 & 0.7621 & 0.7223 \\
\hline$\widehat{\bar{Y}}_{H A}^{(1)}$ & 0.5430 & 0.4661 & 0.4055 & 0.1024 & 0.0913 & 0.0770 & 1.0094 & 0.9322 & 0.8654 \\
\hline$\widehat{\bar{Y}}_{H A}^{(2)}$ & 0.5387 & 0.4646 & 0.4051 & 0.1025 & 0.0906 & 0.0774 & 1.0246 & 0.9345 & 0.8645 \\
\hline$\widehat{\bar{Y}}_{H A}^{(3)}$ & 0.5361 & 0.4689 & 0.4108 & 0.1026 & 0.0910 & 0.0771 & 1.0217 & 0.9388 & 0.8603 \\
\hline$\widehat{\bar{Y}}_{H A}^{(4)}$ & 0.5305 & 0.4658 & 0.4042 & 0.1030 & 0.0914 & 0.0766 & 1.0184 & 0.9236 & 0.8598 \\
\hline$\widehat{\bar{Y}}_{H A}^{(5)}$ & 0.5353 & 0.4633 & 0.4062 & 0.1029 & 0.0912 & 0.0771 & 1.0113 & 0.9283 & 0.8589 \\
\hline$\widehat{\bar{Y}}_{H A}^{(6)}$ & 0.5400 & 0.4706 & 0.4057 & 0.1024 & 0.0914 & 0.0767 & 1.0237 & 0.9407 & 0.8521 \\
\hline$\widehat{\bar{Y}}_{H A}^{(7)}$ & 0.5311 & 0.4678 & 0.4061 & 0.1024 & 0.0904 & 0.0776 & 1.0153 & 0.9345 & 0.8636 \\
\hline$\widehat{\bar{Y}}_{H A}^{(8)}$ & 0.5373 & 0.4666 & 0.4083 & 0.1027 & 0.0915 & 0.0769 & 1.0172 & 0.9308 & 0.8786 \\
\hline$\widehat{\bar{Y}}_{H A}^{(9)}$ & 0.5368 & 0.4658 & 0.4054 & 0.1021 & 0.0911 & 0.0767 & 1.0168 & 0.9285 & 0.8590 \\
\hline$\widehat{\bar{Y}}_{H A}^{(10)}$ & 0.5462 & 0.4721 & 0.4071 & 0.1039 & 0.0910 & 0.0773 & 1.0277 & 0.9446 & 0.8663 \\
\hline$\widehat{\bar{Y}}_{P 1}$ & 0.2253 & 0.1965 & 0.1880 & 0.0744 & 0.0697 & 0.0619 & 0.7530 & 0.7232 & 0.6876 \\
\hline$\widehat{\bar{Y}}_{P 2}$ & 0.2347 & 0.1769 & 0.1574 & 0.0834 & 0.0767 & 0.0669 & 0.7210 & 0.6971 & 0.6664 \\
\hline
\end{tabular}


Table 5. Percentage Relative Efficiencies (PRE) of estimators with respect to $\widehat{\bar{Y}}$ based on simulation study.

\begin{tabular}{|c|c|c|c|c|c|c|c|c|c|}
\hline \multirow[b]{2}{*}{ Estimators } & \multicolumn{3}{|c|}{ Population 1} & \multicolumn{3}{|c|}{ Population 2} & \multicolumn{3}{|c|}{ Population 3} \\
\hline & $n=12$ & $n=14$ & $n=16$ & $n=180$ & $n=200$ & $n=230$ & $n=290$ & $n=310$ & $n=330$ \\
\hline$\widehat{\bar{Y}}$ & 100.000 & 100.000 & 100.000 & 100.000 & 100.000 & 100.000 & 100.000 & 100.000 & 100.000 \\
\hline$\widehat{\bar{Y}}_{R E G}$ & 766.586 & 749.207 & 729.755 & 1206.283 & 1193.833 & 1178.324 & 676.225 & 618.575 & 556.940 \\
\hline$\widehat{\bar{Y}}_{H S 1}$ & 987.347 & 913.926 & 858.046 & 1347.447 & 1313.015 & 1274.462 & 734.719 & 678.189 & 617.416 \\
\hline$\widehat{\bar{Y}}_{H S 2}$ & 812.755 & 785.375 & 758.909 & 1234.139 & 1217.667 & 1197.828 & 696.543 & 639.131 & 577.600 \\
\hline$\widehat{\bar{Y}}_{H S 3}$ & 1098.132 & 990.408 & 914.524 & 1411.218 & 1365.695 & 1315.974 & 753.201 & 697.281 & 637.084 \\
\hline$\widehat{\bar{Y}}_{J I 1}$ & 888.710 & 842.743 & 803.538 & 1279.251 & 1255.959 & 1228.712 & 740.498 & 683.856 & 622.881 \\
\hline$\widehat{\bar{Y}}_{J I 2}$ & 1382.002 & 1165.650 & 1032.976 & 1502.994 & 1440.106 & 1372.636 & 898.397 & 850.142 & 798.285 \\
\hline$\widehat{\bar{Y}}_{H A}^{(1)}$ & 908.289 & 868.816 & 833.734 & 1272.931 & 1257.712 & 1236.909 & 722.773 & 663.921 & 600.093 \\
\hline$\widehat{\bar{Y}}_{H A}^{(2)}$ & 907.008 & 869.154 & 832.204 & 1269.818 & 1254.615 & 1234.438 & 744.068 & 723.019 & 717.034 \\
\hline$\widehat{\bar{Y}}_{H A}^{(3)}$ & 905.622 & 866.220 & 839.286 & 1261.567 & 1244.772 & 1233.626 & 736.168 & 722.732 & 714.374 \\
\hline$\widehat{\bar{Y}}_{H A}^{(4)}$ & 905.313 & 849.367 & 834.382 & 1263.093 & 1260.664 & 1242.487 & 733.023 & 725.365 & 710.147 \\
\hline$\widehat{\bar{Y}}_{H A}^{(5)}$ & 903.575 & 864.829 & 836.822 & 1262.015 & 1259.152 & 1240.036 & 732.665 & 716.237 & 707.388 \\
\hline$\widehat{\bar{Y}}_{H A}^{(6)}$ & 911.161 & 868.626 & 832.772 & 1268.188 & 1250.123 & 1245.323 & 730.110 & 724.998 & 717.704 \\
\hline$\widehat{\bar{Y}}_{H A}^{(7)}$ & 897.334 & 865.880 & 848.572 & 1262. 963 & 1252.886 & 1244.779 & 726.805 & 717.746 & 713.321 \\
\hline$\widehat{\bar{Y}}_{H A}^{(8)}$ & 904.338 & 862.441 & 840.217 & 1259. 486 & 1253.556 & 1240.967 & 733.072 & 719.673 & 714.668 \\
\hline$\widehat{\bar{Y}}_{H A}^{(9)}$ & 907.359 & 878.727 & 838.201 & 1281.329 & 1251.296 & 1234.161 & 729.270 & 724.919 & 708.541 \\
\hline$\widehat{\bar{Y}}_{H A}^{(10)}$ & 894.414 & 853.038 & 839.442 & 1263.584 & 1247.958 & 1232.534 & 725.638 & 715.839 & 707.742 \\
\hline$\widehat{\bar{Y}}_{P 1}$ & 3258.479 & 1952.065 & 1524.815 & 1748.603 & 1642.411 & 1536.096 & 937.697 & 889.288 & 836.048 \\
\hline$\widehat{\bar{Y}}_{P 2}$ & 3055.020 & 1903.397 & 1464.315 & 1561.014 & 1493.770 & 1423.147 & 968.348 & 922.812 & 872.949 \\
\hline
\end{tabular}

peting estimators for all three data sets used in our study;

- From Table 5, the simulation study again reveals that $\widehat{\bar{Y}}_{P 1}$ and $\widehat{\bar{Y}}_{P 2}$ have maximum gain in PREs than all other estimators under competition. This phenomenon is observed in all populations under study.

Therefore, the above findings confirmed that the proposed estimators outperformed the competitors under study.

\section{Concluding remarks}

This study considered the improved estimation of finite population mean under Simple Random Sampling Without Replacement (SRSWOR). Efficient utilization of auxiliary information can play a vital role in this regard. Therefore, the purpose was achieved by using the original information of the auxiliary variable and the ranks of auxiliary variable. Some new differencetype-exponential estimators based on the above idea of dual auxiliary information were proposed. Mathematical properties including bias, Mean Square Error (MSE), and minimum MSE of the proposed estimators were derived up to the first degree of approximation.
To assess the potentiality of the proposed estimators over the competing estimators, real data analysis and simulation study were carried out. Three natural data sets were used for the empirical study. The outcome of this comparison indicated that the proposed estimators were more efficient and less biased than the traditional and other well-known existing estimators. Thus, the researchers are encouraged to use the proposed estimators for estimating the population mean under SRSWOR. The present work could be extended to estimate: (1) The finite population mean under other sampling designs like stratified random sampling and two-phase sampling, etc.; (2) Other unknown finite population parameters including median, variance, proportions, etc.; and (3) Population mean of a sensitive variable in the presence of non-sensitive auxiliary information.

\section{References}

1. Haq, A., Khan, M., and Hussain, Z. "A new estimator of finite population mean based on the dual use of the auxiliary information", Communications in StatisticsTheory and Methods, 46(9), pp. 4425-4436 (2017).

2. Kadilar, C. and Cingi, H. "Ratio estimators in simple 
random sampling", Applied Mathematics and Computation, 151, pp. 893-902 (2004).

3. Kadilar, C. and Cingi, H. "Improvement in estimating the population mean in simple random sampling", Applied Mathematics Letters, 19, pp. 75-79 (2006a).

4. Gupta, S. and Shabbir, J. "On improvement in estimating the population mean in simple random sampling", Journal of Applied Statistics, 35(5), pp. 559566 (2008).

5. Grover, L.K. and Kaur, P. "An improved estimator of the finite population mean in simple random sampling", Model Assisted Statistics and Applications, 6(1), pp. 47-55 (2011).

6. Grover, L.K. and Kaur, P. "A generalized class of ratio type exponential estimators of population mean under linear transformation of auxiliary variable", Communications in Statistics-Simulation and Computation, 43, pp. 1552-1574 (2014).

7. Singh, H.P. and Solanki, R.S. "An efficient class of estimators for the population mean using auxiliary information", Communications in Statistics-Theory and Methods, 42, pp. 145-163 (2013).

8. Haq, A. and Shabbir, J. "Improved exponential type estimators of finite population mean under complete and partial auxiliary information", Hacettepe Journal of Mathematics and Statistics, 43(6), pp. 1079-1093 (2014).

9. Shabbir, J., Haq, A., and Gupta, S. "A new differencecum-exponential type estimator of finite population mean in simple random sampling", Revista Colombiana de Estadistica, 37(1), pp. 199-211 (2014).

10. Ekpenyong, E.J. and Enang, E.I. "Efficient exponential ratio estimator for estimating the population mean in simple random sampling", Hacettepe Journal of Mathematics and Statistics, 44(3), pp. 689-705 (2015).

11. Khan, S.A., Ali, H., Manzoor, S., et al. "A class of transformed efficient ratio estimators of finite population mean", Pakistan Journal of Statistics, 31(4), pp. 353-362 (2015).

12. Solanki, R.S. and Singh, H.P. "The improvement over regression method of estimation of finite population mean in survey sampling", Journal of Advanced Computing, 4(3), pp. 101-111 (2015).

13. Srisodaphol, W., Kingphai, K., and Tanjai, N. "New ratio estimators of a population mean using one auxiliary variable in simple random sampling", Chiang Mai Journal of Science, 42(2), pp. 523-527 (2015).

14. Singh, H.P. and Pal, S.K. "A class of exponential-type ratio estimators of a general parameter", Communications in Statistics- Theory and Methods, 46(8), pp. 3957-3984 (2017).

15. Singh, H.P., Pal, S.K., and Solanki, R.S. "A new class of estimators of finite population mean in sample surveys", Communications in Statistics-Theory and Methods, 46(6), pp. 2630-2637 (2017).
16. Irfan, M., Javed, M., and Lin, Z. "Improved estimation of population mean through known conventional and non-conventional measures of auxiliary variable", Iranian Journal of Science and Technology, Transactions A: Science, 43(4), pp. 1851-1862 (2019).

17. Irfan, M., Javed, M., and Lin, Z. "Efficient ratiotype estimators of finite population mean based on correlation coefficient", Scientia Iranica: Transactions on Industrial Engineering (E), 25(4), pp. 2361-2372 (2018).

18. Javed, M., Irfan, M., and Pang, T. "Hartley-Ross type unbiased estimators of population mean using two auxiliary variables", Scientia Iranica: Transactions on Industrial Engineering (E), 26(6), pp. 3835-3845 (2019).

19. Bahl, S. and Tuteja, R.K. "Ratio and product type exponential estimator", Information and Optimization Sciences, XII(1), pp. 159-163 (1991).

20. Shabbir, J. and Gupta, S. "On estimating finite population mean in simple and stratified random sampling", Communications in Statistics- Theory and Methods, 40(2), pp. 199-212 (2011).

21. Singh, R.K. and Singh, G. "A class of estimators with estimated optimum values in sample surveys", Statistics and Probability Letters, 2, pp. 319-321 (1984).

22. Vishwakarmar, G.K. and Kumar, M. "A general family of dual to ratio-cum-product estimators of population mean in simple random sampling", Chilean Journal of Statistics, 6(2), pp. 69-79 (2015).

23. Rao, J.N.K., Kovar, J.G., and Mantel, H.J. "On estimating distribution function and quantiles from survey data using auxiliary information", Biometrika, 77, pp. 365-375 (1990).

24. Silva, N.P.L.D. and Skinner, C.J. "Estimating distribution functions with auxiliary information using poststratification", Journal of Official Statistics, 11, pp. 277-294 (1995).

25. Nidhi, B.V.S., Sisodia, Singh, S., and Singh, S.K. "Calibration approach estimation of the mean in stratified sampling and stratified double sampling", Communications in Statistics-Theory and Methods, 46(10), pp. 4932-4942 (2017).

26. Singh, R. and Mangat, N.S. "Elements of survey sampling", Norwell, MA: Kluwer Academic Publishers (1996).

27. Kadilar, C. and Cingi, H. "Ratio estimators for the population variance in simple and stratified random sampling", Applied Mathematics and Computation, 173(2), pp. 1047-1059 (2006b).

\section{Biographies}

Muhammad Irfan obtained his PhD degree in Statistics at Zhejiang University, Hangzhou, China in 2018. He holds the position of Assistant Professor at the Department of Statistics, Government College University, 
Faisalabad Pakistan. He has more than 20 research publications in well-reputed journals. His areas of interest are sampling theory, probability distributions, and time series analysis.

Maria Javed obtained her $\mathrm{PhD}$ degree in Statistics at Zhejiang University, Hangzhou, China in 2019. She has been working as an Assistant Professor at the Department of Statistics, Government College University, Faisalabad Pakistan. She has about 15 refereed publications now and has guided research students at all levels of the curriculum. Her research interests include sampling theory and probability distributions.

Sajjad Haider Bhatti received his PhD in Applied Statistics and Econometrics from University of Dijon, France. He received his MSc from the Department of Statistics, Bahauddin Zakariya University, Multan, Pakistan. He is currently working as an Assistant Professor at the Department of Statistics, Government College University, Faisalabad, Pakistan. His Research interests include regression diagnostics, modified estimators, sampling theory, and statistical applications in varying fields. 